\title{
Estimation des rendements et de la rentabilité économique de production de trois cultures : le sorgho, le niébé et la dolique à Djirataoua (Maradi - République du Niger)
}

\author{
Mahamadou MALAM ABDOU1, Salissou ISSA ${ }^{1}$, Abdou DAN GOMMA¹, Adama SOW ${ }^{2}$, Germain Jérôme \\ SAWADOGO2 \\ 1. Institut National de Recherches Agronomiques du Niger, BP 429 Niamey-Niger \\ 2. École Inter-états des Sciences et Médecine Vétérinaires - BP 5077 Dakar - Fann - Dakar (Sénégal) \\ * Auteur correspondant : Email : mamalam1@yahoo.fr Tél : (+227) 9688 86 75, (+227) 90011322
}

Original submitted in on 20 June 2017. Published online at www.m.elewa.org on 30 September 2017 https://dx.doi.org/10.4314/jab.v117i1.1

\begin{abstract}
RESUME
Objectif : Un essai de production du sorgho, du niébé et de la dolique a été conduit en 2014 sur le périmètre irrigué de Djirataoua. II vise à estimer les rendements en fourrage et en grain, déterminer la composition chimique du fourrage et évaluer la rentabilité économique de production des trois cultures.

Méthodologie et résultats : L'essai est un bloc complet randomisé de trois traitements en quatre répétitions. Les données sont collectées sur le cycle végétatif des cultures, le coût des intrants utilisés, les coûts de la main d'œuvre pour les travaux et les prix des différents produits et sous produits obtenus après la récolte. Les résultats obtenus sont : Pour le fourrage, les rendements sont de 8,6 00,7 tonnes ha-1 de MS pour le sorgho, $5,6 \pm 0,74$ tonnes ha-1 de MS pour le niébé et $4 \pm 0,74$ tonnes ha-1 de MS pour la dolique. Les rendements grains sont de $3,4 \pm 0,65$ tonnes ha-1 pour le sorgho, 1,2 $\pm 0,65$ tonnes ha-1 ${ }^{-1}$ pour le niébé et celui de la dolique est nul. L'analyse chimique de la biomasse a montré que les trois cultures ont une teneur en MS comparable. Par contre, le niébé et la dolique sont plus riches en MAT que le sorgho : la teneur en MAT du sorgho est de $8 \%$, elle est le double pour le niébé et le triple pour la dolique. L'analyse économique de la production a donnée une marge brute de 730500 FCFA pour le niébé, 339200 FCFA pour le sorgho et 46300 FCFA pour la dolique.

Conclusion et application des résultats : Cette étude montre que les cultures à double fin, du sorgho du niébé et de la dolique donnent un rendement en fourrage important moyennant une densité forte, une bonne fertilisation des sols et un bon entretien cultural. Cela constitue un remède au déficit fourrager chronique au Niger et permet d'accroître la productivité du cheptel et le revenu des producteurs.

Mots clés : sorgho, niébé, dolique, fourrage, rentabilité, République du Niger
\end{abstract}



de trois cultures : le sorgho, le niébé et la dolique à Djirataoua (Maradi - République du Niger)

Estimated yields and economic profitability for the production of three crops : sorghum, cowpea and dolic to Djirataoua (Maradi, Niger Republic)

\begin{abstract}
Objectives : Production test on sorghum, cowpeas and dolic peas was conducted in 2014 on an irrigated perimeter of Djirataoua. It aimed to estimate forage and grain yields, chemical analysis of forage and evaluate the economic profitability of the three crops.

Methodology and results : The test was set in a randomized complete block design with three plots in four replicates. The basic plot had a size of $5 \mathrm{~m} \times 100 \mathrm{~m}$. The agronomic data was collected on the growth cycle of crops, while economic data covered the cost of inputs, labor costs for the work and prices of various products and by-products obtained at harvest. Fodder and grain yields were evaluated. For fodder, the results obtained were $8.6 \pm 0.7$ tons ha- $1 \mathrm{DM}, 5.6 \pm 0.74$ tons ha- $1 \mathrm{DM}$ and $4 \pm 0.74$ tons ha-1 DM respectively for Sorghum, cowpea and dolic peas. Grain yields were $3.4 \pm 0.65$ tons ha- 1 for sorghum, $1.2 \pm 0.65$ tons ha- 1 for cowpea, and dolic peas yield is zero because it is a photosensitive plant. Chemical analysis of the biomass showed that the three crops had a comparable DM content, whereas cowpeas and dolic peas were richer in total nitrogenous matter (TNM) than sorghum. The content of TNM is $8 \%$ for sorghum; it is double in cowpea and triple in dolic peas.

Conclusion and applications of results: This study shows that the dual-purpose crops, sorghum and cowpea provide an important forage yield meaning a high density and good soil fertility and good crop maintenance. This can be a cure for the chronic fodder deficit in Niger and will increase livestock productivity and farmers' income.
\end{abstract}

Keywords: sorghum, cowpea, dolic, forage, profitability, Djirataoua, Republic of Niger

\section{INTRODUCTION}

La disponibilité en quantité et en qualité du fourrage est la contrainte majeure dans l'alimentation du bétail au sahel en général (Hiernaux et al., 2016). Au Niger, cette alimentation est basée essentiellement sur le pâturage naturel et les résidus de cultures (Camara, 2010). De l'analyse du bilan fourrager de 2000 à 2014, il ressort qu'en moyenne une année sur deux est déficitaire avec des valeurs extrêmes allant de 1387964 tonnes de MS en 2002 à 16137329 tonnes de MS en 2009 (DDP/ DGPIA, 2015). Dans la région de Maradi, avec la pression démographique en particulier au sud, les jachères ont disparues. Les quelques enclaves laissées comme aires de pâturage pour les animaux sont dégradées et envahies par Sida cordifolia, une espèce de plante non appétée par les animaux

\section{MATERIEL ET METHODE}

Site expérimental: L'essai a été implanté sur le périmètre irrigué de la commune de Djirataoua. Ce périmètre est localisé à $7 \mathrm{kms}$ au Sud-est de la ville de Maradi entre $13^{\circ} 20$ à $13^{\circ} 25$ de latitude Nord et $07^{\circ} 10$ à $07^{\circ} 11$ de longitude Est. Les sols sont développés sur des
(Mahamane, 2011). Le déficit fourrager est chronique depuis 2008 (DDP/ DGPIA, 2015) malgré les quantités de pluies qui tombent chaque année dans cette zone, entrainant des répercussions négatives sur la productivité des animaux. L'une des solutions pouvant contribuer à la réduction de ce déficit et améliorer la qualité fourragère est la promotion de la culture fourragère en utilisant des espèces végétales adaptées au milieu et l'amélioration de la production des résidus de cultures. Ainsi la présente étude a été conduite à Djirataoua avec comme objectif, l'analyse des rendements et de la rentabilité économique de la production de trois cultures, le sorgho, le niébé et la dolique.

alluvions de grande diversité texturale allant de la texture fine (argile) à la texture grossière (sable, sablo limoneux) suivant leur position topographique (ONAHA, 1980). 

de trois cultures : le sorgho, le niébé et la dolique à Djirataoua (Maradi - République du Niger)

Matériel végétal : Le matériel végétal est composé de trois cultures: le sorgho, le niébé et la dolique. Le sorgho utilisé est la variété SEPON-82 (Sorghum Ellit Preliminary Observation Nursery). Elle a été introduite au Niger en 1983. C'est une variété originaire de « International Crops Research Institute for the Semi-Arid Tropics » (ICRISAT) en Inde et sélectionnée d'un croisement avec trois parents élites (GPR148, E35-1 et CS3541). La variété SEPON-82 est une variété améliorée qui dispose un fort potentiel en production de graine (2,5 à 4 tonnes/ha) et une bonne qualité fourragère car les feuilles restent vertes jusqu'à la maturité et les tiges sont sucrées. Pour le niébé, nous avons utilisé la variété locale « $L A K A D E$ » qui est très appréciée sur le périmètre de Djirataoua pour sa production fourragère. La dolique (Dolichos lablab) a été introduite au Niger dans les années 1960 avec le programme d'expérimentation des cultures fourragères, conduit par les institutions internationales notamment Institut de Recherche Agronomique Tropicale (IRAT) et depuis lors elle n'a connu aucune amélioration dans le pays. La dolique est cultivée en culture de relais dans les vallées sèches. Une fois semée en début du mois de septembre, elle reçoit un seul sarclage à la levée. Elle poursuit son cycle végétatif en période froide de l'année (octobre novembre et décembre) où elle bénéficie de l'humidité résiduel du sol.

\section{Méthode}

Dispositif expérimental : Le dispositif expérimental est un bloc complet randomisé à quatre répétitions avec des parcelles élémentaires de $5 \mathrm{~m}$ x $100 \mathrm{~m}$ (figure 1). La superficie globale de l'essai était de $6400 \mathrm{~m}^{2}$.

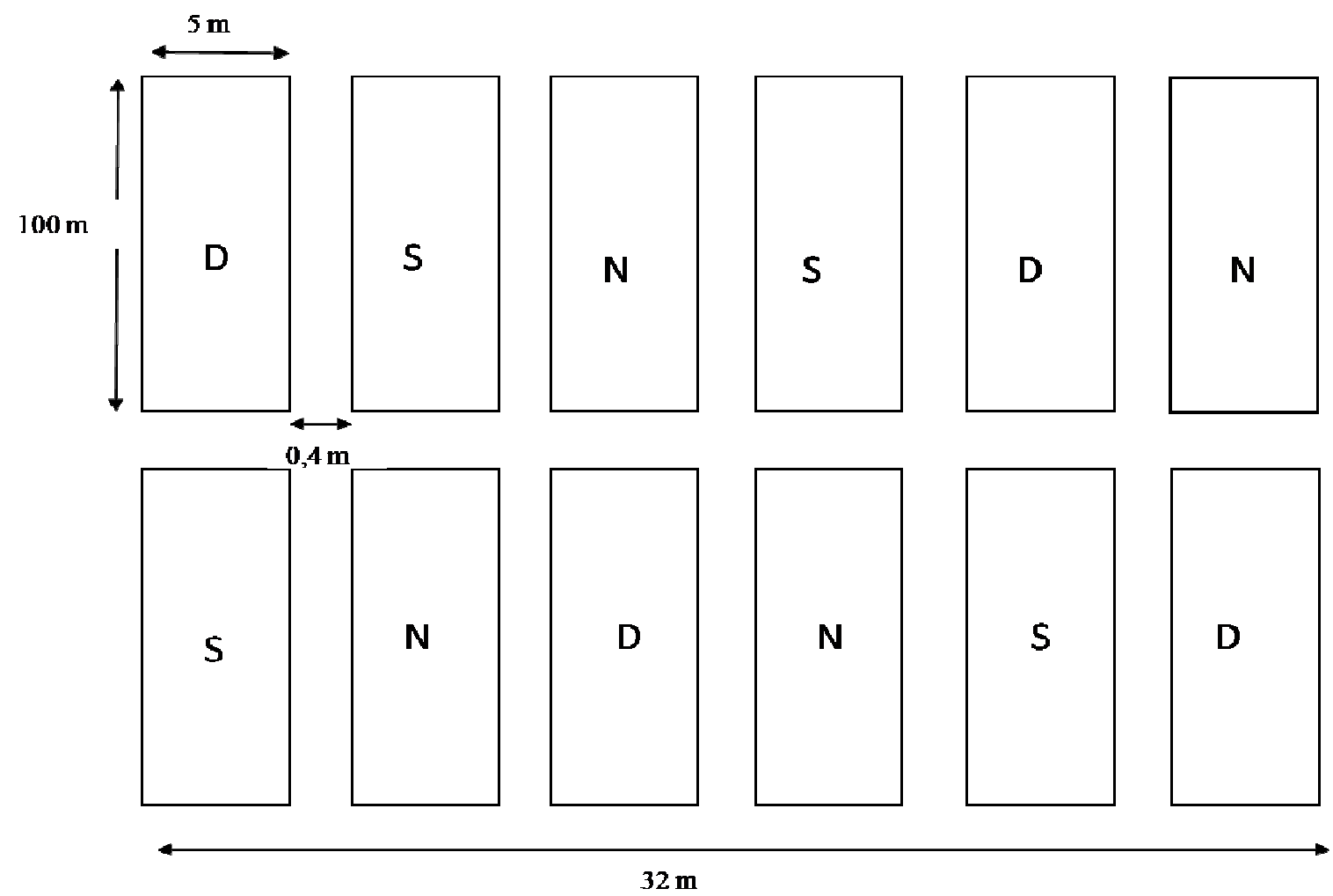

$\mathrm{D}=$ Dolique $; \mathrm{S}=$ Sorgho $; \mathrm{N}=$ Niébé.

Figure 1: Dispositif expérimental de l'essai sur le périmètre irrigué de Djirataoua

Installation de l'essai : L'expérimentation a été conduite durant la saison d'hivernage de 2014. Après le labour avec une charrue à soc, il a été appliqué sur l'ensemble de la parcelle expérimentale de l'engrais NPK de composition chimique 15-15-15 à la dose de $100 \mathrm{~kg} / \mathrm{ha}$ et du fumier à la dose de 2 tonnes /ha. Le semis a eu lieu le $12 / 08 / 2014$ avec un écartement de $0,8 \mathrm{~m}$ x 0,5 $\mathrm{m}$ pour le sorgho et 0,8 $\mathrm{m} \times 1 \mathrm{~m}$ pour le niébé et la dolique soit une densité au semis de 25000 poquets / ha pour le sorgho et 12500 poquets / ha pour le niébé et la dolique. Les différentes opérations culturales d'entretien composées d'un démariage, deux sarclages et deux traitements phytosanitaires sur les légumineuses sont faites normalement. 


\section{Abdou et al., J. Appl. Biosci. 2017 Estimation des rendements et de la rentabilité économique de production}

de trois cultures : le sorgho, le niébé et la dolique à Djirataoua (Maradi - République du Niger)

\section{Collecte des données}

Sol: Deux échantillons composites du sol sur une profondeur de 0 à $20 \mathrm{~cm}$ ont été prélevés sur la parcelle expérimentale avant les apports de la fumure organique et minérale pour une analyse physico-chimique. L'analyse a porté sur la granulométrie et la composition chimique $(\mathrm{pH}, \mathrm{C}, \mathrm{N}, \mathrm{P}, \mathrm{Ca})$. La granulométrie a été faite par la méthode de BOUYOUCOS. Le $\mathrm{PH}_{\text {eau }}$ est déterminé selon le rapport $1 / 2,5$ ( $10 \mathrm{~g}$ de sol pour $25 \mathrm{ml}$ d'eau distillée). La matière organique (MO) totale est obtenue par le dosage du carbone organique par la méthode de WALKLEY $B L A C K$, tandis que la détermination de l'azote $(\mathrm{N})$ total a été faite par la méthode macro-KJELDAHL. Les bases échangeables $\left(\mathrm{K}^{+}, \mathrm{Na}^{+}, \mathrm{Ca}^{++}\right.$et $\left.\mathrm{Mg}^{++}\right)$sont déterminées par photométrie à flamme pour $\mathrm{K}+$ et $\mathrm{Na}+$ et par spectrophotomètre à absorption atomique Perkin Elmer 2380 pour le $\mathrm{Ca}^{++}$et le $\mathrm{Mg}^{++}$.

Cultures : II s'agissait de la durée du cycle végétatif, les mesures des rendements en fourrage et en grain et la composition chimique de la biomasse des trois cultures. Les données ont été collectées sur trois carrés de $5 \mathrm{~m} \times 2$ $\mathrm{m}$ placés à équidistance au niveau de chaque parcelle élémentaire. Sur le cycle végétatif, les observations ont été effectuées sur les paramètres agronomiques comprenant : date de levée, densité et nombre de jours au stade $50 \%$ ramification ou montaison de la culture, nombre de jours à $50 \%$ floraison ou épiaison, nombre de jours à $100 \%$ maturité. Les rendements en fourrage des trois cultures ont été évalués, de même que les rendements en grain sur le niébé et le sorgho à 100 jours après semis (JAS) correspondant au stade de maturité de ces cultures. Le rendement en grain de la dolique était nul à ce stade à cause de la photosensibilité de la plante. Des échantillons du fourrage de chaque culture ont été prélevés à 100 JAS pour la détermination de la composition chimique. Les éléments analysés sont la matière sèche (MS), la matière azotée totale (MAT) ou protéine brute, la matière minérale (MM), la cellulose brute $(C B)$, les matières grasses ou extrait éthéré (EE) et les éléments minéraux majeurs notamment le phosphore $(\mathrm{P})$ et le calcium ( $\mathrm{Ca}$ ). La MS a été déterminée après séchage à l'étuve à $105^{\circ} \mathrm{C}$ jusqu'à poids constant. La MM ou cendres a été obtenue par passage de l'échantillon sec dans un four à moufle à $550^{\circ} \mathrm{C}$ pendant 5 à $7 \mathrm{~h}$ jusqu'à l'obtention de cendres blanches ou grises, alors que la MAT ou protéine brute, a été déterminée par la méthode de $\mathrm{KJELDAH}(6,25 \times \% \mathrm{~N})$. Pour la CB, elle a été déterminée par la méthode de WEENDE qui est l'une des méthodes la plus utilisée pour doser les constituants des parois cellulaires des végétaux. Quant aux matières grasses (MG) ou extrait éthéré $(E E)$, elles ont été obtenues par utilisation de solvant organique. La détermination de la teneur en matières grasses est basée sur la solubilisation de ces matières en acides gras dans un solvant approprié et volatil, en l'occurrence l'éther de pétrole. Les éléments minéraux majeurs à savoir le phosphore $(\mathrm{P})$ et le calcium $(\mathrm{Ca})$ contenu dans les végétaux ont été dosés par le même principe du dosage de ces éléments dans le sol.

Données économiques: Pour calculer la rentabilité économique de la production des trois cultures, il a été procédé à une évaluation à l'hectare des charges et des recettes. Ainsi plusieurs types de données ont été collectées : Pour les charges il s'agit de :

- Coûts des intrants (engrais, fumier, semences, fongicide et pesticide) ;

- Coût des travaux (du labour de la parcelle jusqu'au transport de la production) ;

- $\quad$ Et d'autres charges comme la location terrain, l'achat des sacs de conditionnement.

S'agissant des recettes, les données ont concerné les prix de vente de la production graine et du fourrage des cultures. Les différents prix ont été collectés lors d'une enquête réalisée dans la commune de Djirataoua et auprès des services étatiques. Ainsi le prix de l'engrais était de $270 \mathrm{FCFA} / \mathrm{kg}(0.6 \mathrm{U} \$ / \mathrm{kg})$ pour l'Urée, $280 \mathrm{~F} / \mathrm{kg}$ (0.62 U\$ $/ \mathrm{kg}$ ) pour le NPK (15-15-15), le pesticide (TITAN $25 \mathrm{EC})$ était à $8000 \mathrm{FCFA} / \mathrm{L}(17.8 \mathrm{U} \$ / \mathrm{L})$ et le fongicide 200 FCFA (0,4 U\$) /sachet de 100g. Le prix du fumier s'élevait à $40 \mathrm{FCFA} / \mathrm{kg}$ soit moins de $0.1 \mathrm{U} \$$ à la station avicole de Maradi. Les semences du sorgho ont été achetées auprès de l'Unité Semencière de l'INRAN à 2000 FCFA (4.4 U\$)/kg, tandis que les semences du niébé et de la dolique ont été achetées sur le marché local à raison de 750 FCFA (1.7 U\$)/kg. La main d'œuvre pour les différents travaux agricoles était à 2000 FCFA (4.4 U\$)/homme -jour pour l'homme et 1000 FCFA (2.2 U\$) pour la femme alors que le labour coûtait 5000 FCFA (11.1 U\$)/jour de travail d'une paire de bœuf. Quant au transport du champ au village des tiges de sorgho et fanes de niébé ou de la dolique, le prix a été estimé à 15 FCFA (0.03 U\$) pour un fagot de tiges de sorgho de 10 $\mathrm{kg}$ en moyenne (soit 1,5 FCFA $/ \mathrm{kg}$ ) et à 10 FCFA $(0.02$ U\$) pour une botte de fanes de niébé du même poids (soit $1 \mathrm{FCFA} / \mathrm{kg}$ ). Les prix des produits et sous-produits agricoles, ont été collectés sur les marchés de la commune de Djirataoua en période de récolte (Tableau 1). 

de trois cultures : le sorgho, le niébé et la dolique à Djirataoua (Maradi - République du Niger)

Tableau 1: Prix moyens des produits et sous-produits agricoles sur les marchés de la commune rurale de Djirataoua au Niger.

\begin{tabular}{l|l}
\hline Produits /sous-produits & Prix moyen (FCFA/kg) \\
\hline Niébé grain & $313 \pm 10$ \\
Sorgho grain & $146 \pm 10$ \\
Cosse de niébé & $263 \pm 40$ \\
Glumes de sorgho & $0,00 \pm 0$ \\
Fanes d'arachide & $76 \pm 25$ \\
Fanes de niébé & $84 \pm 26$ \\
Fanes de la dolique & $84 \pm 26$ \\
Tiges de sorgho & $24 \pm 7$ \\
\hline
\end{tabular}

Traitement et analyse statistique des données : Les données collectées ont été saisi au logiciel Excel avant d'être transférées au Genstat pour une analyse de variance pour certaines variables par le General Linear Model (GLM) et la comparaison des moyennes a été faite par le test LSD au seuil de $5 \%$.

\section{RESULTATS}

Caractéristique des sols: Le sol de la parcelle expérimentale a une texture sablo limoneuse et un $\mathrm{pH}$ modérément basique avec une valeur de 7,9. Le taux de

la matière organique (M.O) est de $0,89 \%$ et celui de l'azote est $0,047 \%$. Le sol est modérément lessivé en base $(\mathrm{C} / \mathrm{N}=11)$ (Tableau 2).

Tableau 2 : Caractéristiques physico-chimiques du sol de la parcelle expérimentale à une profondeur de 0 à $20 \mathrm{~cm}$ sur le périmètre irrigué de Djirataoua au Niger.

\begin{tabular}{l|c}
\hline Éléments & Valeurs \\
\hline Argile (\%) & 3,30 \\
Sable Fin (\%) & 75,50 \\
Sable Grossier (\%) & 2,20 \\
Limon (\%) & 18,90 \\
pH eau & 7,90 \\
MO (\%) & 0,89 \\
C total (\%) & 0,52 \\
C/N & 11,00 \\
$\mathrm{~N}$ total (\%) & 0,05 \\
$\mathrm{~K}$ (méq) & 0,57 \\
Mg (méq) & 3,94 \\
$\mathrm{Na}$ (méq) & 0,28 \\
\hline
\end{tabular}

Cycle de développement des cultures: Le cycle végétatif des cultures a été suivi du semis à la récolte (Tableau 3). La levée des trois cultures a eu lieu dans un délai maximal de 6 jours. Elle est plus précoce pour le sorgho ensuite le niébé et enfin la dolique. La densité de la dolique et du niébé au stade ramification est supérieure à celle au stade semis car il y a eu un ressemis pour ces deux cultures sur trois répétitions, alors que celle du sorgho est inférieure à la densité au semis. Les parcelles du sorgho ont atteint $50 \%$ montaison à 31 jours après le semis (JAS), celles du niébé et de la dolique sont à $50 \%$ ramification dans l'intervalle de 37 à 38 JAS. Quant au stade $50 \%$ épiaison pour le sorgho il a été noté à $67 \mathrm{JAS}$, la floraison à $50 \%$ est enregistrée à $75 \mathrm{JAS}$ pour le niébé tandis que le stade début floraison est à $105 \mathrm{JAS}$ pour la dolique. La récolte au stade maturité totale est intervenue dans l'intervalle de 100 à $105 \mathrm{JAS}$ pour le sorgho et le niébé. Pour la dolique, elle est récoltée à $120 \mathrm{JAS}$ sans qu'il ait véritablement la formation des gousses. Le rendement grain obtenu est de 3,4 tonnes/ha pour le sorgho et 1,2 tonne/ha pour le niébé ; aussi nous avons obtenu $800 \mathrm{~kg} \mathrm{ha}^{-1}$ de cosse de niébé après battage. La dolique qui est une culture photosensible a été récoltée sans fructification avec l'arrivée de l'harmattan qui provoquait la chute des feuilles. 

de trois cultures : le sorgho, le niébé et la dolique à Djirataoua (Maradi - République du Niger)

Tableau 3 : Cycle de développement et de productions des cultures du sorgho (Sepon 82), de la dolique et du niébé (Lakadé), au cours de la saison de pluies 2014, sur le périmètre irrigué de Djirataoua au Niger.

\begin{tabular}{l|c|c|c}
\hline Paramètres & Sorgho & Dolique & Niébé \\
\hline Levée (JAS) & $4 \pm 1$ & $6 \pm 1$ & $5 \pm 1$ \\
Montaison à 50\% (JAS) & $31,5 \pm 1,01$ & - & - \\
Ramification à 50\% (JAS) & - & $37 \pm 1$ & $38 \pm 1$ \\
Densité à 50\% montaison ou Ramification (poquets/ha) & $23588 \pm 1691$ & $19583 \pm 1690$ & $13208 \pm 1690$ \\
Épiaison à 50\% (JAS) & $67 \pm 1$ & - & - \\
Floraison à 50\% (JAS) & $100 \pm 1$ & - & $75 \pm 0,4$ \\
Récolte (JAS) & $3,4 \pm 0,7$ & - & $102 \pm 1$ \\
Rendement Grain (tonnes ha-1) & - & - & $1,2 \pm 0,7$ \\
Rendement cosse (tonnes ha-1) & & - & $0,8 \pm 0,4$ \\
\hline
\end{tabular}

JAS = jour après semis

Évaluation des rendements de fourrage des trois cultures : Les rendements en fourrage évalués pour les trois cultures sont rapportés dans le tableau 4. Pour le sorgho, ce rendement était de 36,1 tonnes ha-1 de M.V soit 8,6 $\pm 0,7$ tonnes ha-1 de MS, il est 28,1 tonnes ha-1 de $^{-1}$ MV (5,6 $\pm 0,7$ tonnes ha- 1 de MS) pour le niébé et 22,4 tonnes ha-1 de MV $\left(4 \pm 0,7\right.$ tonnes ha-1 $^{-1}$ de MS) pour la dolique.

Tableau 4 : Rendement en biomasse de trois cultures (sorgho, dolique et niébé) sous régime pluvial sur le périmètre irrigué de Djirataoua au Niger.

\begin{tabular}{c|c|c|c|c|c|c}
\hline \multirow{2}{*}{ Stade de développement } & \multicolumn{4}{|c}{ Rendement (tonne/ha) } \\
\cline { 2 - 7 } & \multicolumn{2}{|c|}{ Sorgho (Sepon 82) } & \multicolumn{2}{|c}{ Dolique } & \multicolumn{2}{c}{ Niébé (Lakadé) } \\
\hline & MV & MS & MV & MS & MV & MS \\
100 JAS (stade de récolte) & $36,1 \pm 3,2$ & $8,6 \pm 0,7$ & $22,4 \pm 3,2$ & $4 \pm 0,7$ & $28,1 \pm 3,2$ & $5,6 \pm 0,7$ \\
\hline
\end{tabular}

$\mathrm{MV}=$ matière verte $; \mathrm{MS}=$ matière sèche

Composition chimique de la biomasse : Les fourrages prélevés à $100 \mathrm{JAS}$ correspondant au stade maturité des graines pour le sorgho et le niébé, avaient un taux en MS de $83 \pm 0,03$ pour le sorgho et $86 \pm 0,34$ pour le niébé et la dolique. Par contre, le niébé et la dolique se montraient plus riches en MAT que le sorgho. En effet, la teneur en MAT est de $8 \%$ dans les tiges de sorgho, elle est le double dans les fanes de niébé et triple dans celles de la dolique (Tableau 5). Pour la teneur en CB et en MM, le niébé était plus riche en $\mathrm{CB}$ que la dolique et le sorgho, alors qu'il est moins riche en MM que ces deux cultures qui elles, ont des valeurs comparables pour la $\mathrm{CB}$ et pour la MM. Concernant la teneur en éléments minéraux majeurs (le phosphore et le calcium), le niébé est plus riche pour ces deux éléments que la dolique et le sorgho qui ont des résultats comparables (Tableau 5).

Tableau 5 : Composition chimique de la biomasse des cultures du sorgho, du niébé et de la dolique selon les deux stades végétatifs.

\begin{tabular}{|c|c|c|c|c|c|c|c|}
\hline \multirow{2}{*}{ Cultures } & \multirow{2}{*}{ MS (\%) } & \multicolumn{4}{|c|}{ Matière sèche } & \multirow{2}{*}{$\mathrm{Ca}$ (ppm) } & \multirow{2}{*}{$P_{\text {tot }}(p p m)$} \\
\hline & & MM (\%) & CB (\%) & MAT(\%) & EE (\%) & & \\
\hline Sorgho & $83 \pm 0,03$ & $6 \pm 0,03$ & $31 \pm 0,02$ & $8 \pm 0,00$ & $0,1 \pm 0,00$ & $226 \pm 0,00$ & $1905 \pm 2,05$ \\
\hline Niébé & $86 \pm 0,34$ & $3 \pm 0,15$ & $43 \pm 0,50$ & $13 \pm 0,79$ & $0,3 \pm 0,05$ & $456 \pm 2,43$ & $808 \pm 3,6$ \\
\hline Dolique & $86 \pm 0,34$ & $10 \pm 0,05$ & $37 \pm 0,35$ & $24 \pm 0,03$ & $0,2 \pm 0,05$ & $336 \pm 0,98$ & $1030 \pm 3,7$ \\
\hline
\end{tabular}

Analyse économique de la production du sorgho, niébé et de la dolique : L'analyse économique a montré une marge nette positive pour les trois cultures (Tableau
6). Cette marge nette de 730500 FCFA/ha pour le niébé est plus élevée, suivie de 339200 FCA pour le sorgho et $46300 \mathrm{FCFA} / \mathrm{ha}$ pour la dolique. Les charges liées à la 


\section{Abdou et al., J. Appl. Biosci. 2017 Estimation des rendements et de la rentabilité économique de production de trois cultures : le sorgho, le niébé et la dolique à Djirataoua (Maradi - République du Niger)}

main d'œuvre sont très élevées, elles occupent de $52 \%$ à $56 \%$ de dépenses totales au niveau des trois cultures. Pour le sorgho, les recettes en produit grain sont plus importantes que celles de tiges, sans lesquelles la marge brute est négative pour la culture (Tableau 6). En revanche pour le niébé, les recettes en fanes sont nettement supérieures à celles des produits grains auxquelles s'ajoutent les recettes provenant des cosses de niébé permettant ainsi de gagner une marge nette très élevée. Quant à la dolique qui avait produit uniquement le fourrage en saison de pluies, elle n'a pas dégagé une marge nette aussi importante que le niébé (Tableau 6).

Tableau 6 : Charge et marge bénéficiaire par hectare de production des cultures de sorgho, niébé et dolique sur le périmètre irrigué de Djirataoua au Niger.

\begin{tabular}{|c|c|c|c|}
\hline Rubriques & Sorgho & Niébé & Dolique \\
\hline \multicolumn{4}{|l|}{ A charges } \\
\hline 1- Intrants (FCFA/ha) (1) & 151200 & 128700 & 128700 \\
\hline 2- Main d'œuvre (FCFA/ha) (2) & 183900 & 181600 & 141000 \\
\hline 3- Autres charges (conditionnement) (3) & 8500 & 11250 & 0 \\
\hline Charge totale (FCFA /ha) (A) : (1) +(2) +(3) & 343600 & 321550 & 269700 \\
\hline \multicolumn{4}{|l|}{ B- Recette } \\
\hline 1- fourrage et cosses niébé (FCFA/ha) (1) & 206400 & 680800 & 336000 \\
\hline 2- grain (sorgho, niébé) (FCFA/ha) (2) & 496400 & 391250 & 0 \\
\hline Total recettes (FCFA/ha) (B) : (1) + (2) & 702800 & 1072050 & 336000 \\
\hline Marge brute (FCFA/ha) (B-A) & 359200 & 750500 & 66300 \\
\hline \multicolumn{4}{|l|}{ C- Amortissement } \\
\hline Location terrain & 20000 & 20000 & 20000 \\
\hline Total $(\mathrm{C})$ & 20000 & 20000 & 20000 \\
\hline Marge nette (FCFA/ha) (B-A-C) & 339200 & 730500 & 46300 \\
\hline
\end{tabular}

\section{DISCUSSION}

Le sol de la parcelle expérimentale est un sol sablolimoneux, modérément lessivé avec un pH légèrement basique. La matière organique et l'azote ont respectivement un taux de $0,89 \%$ et $0,047 \%$. II appartient à la classe des sols hydromorphes de la vallée du Goulbi N' Maradi (Mahaman, 2001, Moussa, 2013). Pour le développement végétatif, la variété du sorgho SEPON-82 a bouclé son cycle dans l'intervalle de 90 à $105 \mathrm{JAS}$ avec un rendement grain de l'ordre de 3 tonnes/ha et un rendement en biomasse de 8,6 tonnes MS/ha. Ceci est conforme aux caractéristiques de cette variété qui sont mentionnées dans le catalogue national des espèces et variétés végétales ( $M / A, 2012)$. Le niébé (LAKADE) a donné un rendement en biomasse de 5,6 tonnes MS/ha et un rendement grain de 1,2 tonne/ha. A travers ces résultats, le niébé (LAKADE) remplissait les caractéristiques des variétés améliorées à double usage pouvant produire jusqu'à 1,5 tonne ha-1 de grain et 2,5 tonnes ha-1 de fanes (Singh et al., 2003) et répondant à l'intégration agriculture-élevage dans les zones semiarides tropicales (Tarawali, 2002 ; Bambara et al., 2008). Ces résultats sont nettement supérieurs à ceux rapportés par Ado Alassan (2014) avec la même variété qui a donné un rendement de $1174 \mathrm{~kg}$ de $\mathrm{MS} \mathrm{ha}^{-1}$ et un rendement grain de $225 \mathrm{~kg} \mathrm{ha}^{-1}$ sur le sol sableux de la station de N'Dounga à Kollo en culture pluviale. Cette différence observée était probablement liée au sol et à la pluviométrie ; les densités de semis étant plus faibles dans nos conditions ( $1 \mathrm{~m} \times 0,8 \mathrm{~m}$ ) que celles de $1 \mathrm{~m} \times 0,4$ $m$ (Ado Alassan, 2014). En revanche, le rendement en biomasse est du même ordre que les rendements des lignées VITA3 (9,3 tonnes ha-1), IT83 S-755-1 (8,2 tonnes ha-1 $\left.^{-1}\right)$, TVX 1948-01 (7,7tonnes ha- $\left.{ }^{-1}\right)$ trouvés par Akundaweni et al., (1991). Pour la dolique, le rendement en biomasse est nettement supérieur à celui du milieu paysan. Cependant on note une absence de la formation des gousses jusqu' à 120 JAS contrairement au milieu paysan. En effet, le rendement en biomasse obtenu est plus important que celui du milieu paysan à cause non seulement de la faible densité utilisée chez les paysans, mais aussi du manque d'entretien (1 seul sarclage pour toute la durée du cycle). Quant au manque de formation de gousse dans nos conditions, il est lié au caractère photosensible de la plante. En effet les paysans sèment la dolique en fin septembre et elle atteint le stade floraison et maturation des gousses en période de jours courts qui s'étale de Novembre à Février au Niger. En considérant que la production primaire de la matière 


\section{Abdou et al., J. Appl. Biosci. 2017 Estimation des rendements et de la rentabilité économique de production de trois cultures : le sorgho, le niébé et la dolique à Djirataoua (Maradi - République du Niger)}

sèche du sorgho est consommée par le bétail à $35 \%$, et celle du niébé et de la dolique à $60 \%$ (Hadi, 2000) d'une part et en supposant qu'une UBT consomme $6,25 \mathrm{~kg}$ MS/jour, la quantité du fourrage disponible par hectare permettrait de nourrir environ 1,3 UBT/an pour le sorgho, 1,1 UBT/an pour la dolique et 1,5 UBT/an pour le niébé. Par contre selon Karimou et Atikou (1998) qui ont rapporté que $79,5 \%$ de la production primaire du sorgho et $86,3 \%$ de celle du niébé sont disponibles pour la supplémentation des animaux, la capacité en charge de 1 ha peut atteindre 3 UBT/an, 1,5 UBT/an et 2,1 UBT/an respectivement pour le sorgho, la dolique et le niébé. L'analyse bromatologique de la biomasse, montre qu'à maturité, le sorgho a un taux de MAT de $8 \%$ situé au seuil du taux critique d'utilisation des protéines brute estimé à $7 \%$ (Van Soest, 1982) en deçà duquel l'activité de la microflore du rumen des ruminants domestiques diminue, tandis que le niébé et la dolique ont un taux nettement supérieur au seuil respectivement $13 \%$ et $24 \%$. Cela montre l'importance de l'association du fourrage du sorgho avec celui du niébé ou de la dolique dans l'alimentation animale pour une complémentation en matière azotée. Sur le plan économique, les résultats montrent que la production du fourrage du sorgho seule n'est pas rentable, il faut qu'elle soit accompagnée de la production graine. Cette situation provient du faible coût

\section{CONCLUSION}

Les cultures du sorgho (Sepon 82), du niébé (Lakadé) et de la dolique ont des rendements importants en fourrage moyennant une densité forte, et un bon entretien des cultures. La production fourragère est rentable à condition qu'il ait un prix rémunérateur du fourrage même à la récolte. Nous encourageons la production de ces cultures

REMERCIEMENTS : L'équipe adresse ses sincères remerciements au projet AusAID/COAF/WECARD pour avoir financé cette étude. Elle remercie également la

\section{RÉFÉRENCE BIBLIOGRAPHIQUE}

Ado Alassan N., 2014. Analyse technico-économique de la production de quatre (4) variétés fourragères de niébé dans la station agronomique de I'INRAN et évaluation de la valeur alimentaire de ses fanes dans le laboratoire, Mémoire Master en Productions Animales et Développement Durable (PADD), Spécialité : Économie et Politiques d'Élevage (EPE), EISMV Dakar, 48p Akundabweni L.S., Paul C.P., Singh B.B., 1991. Évaluation de lignées d'élite de niébé pour un des tiges de sorgho sur le marché en période de récolte. Par contre, la production du fourrage du niébé et de la dolique est rentable. La production graine et celle des cosses du niébé ou de la dolique devient un gain supplémentaire. C'est pourquoi sur le périmètre de Djirataoua et le long de la vallée du Goulbi N'Maradi, les producteurs s'adonnent à la pratique de ces cultures. Dabat et al. (2012) ont rapporté pour une densité de 5000 pieds/ha (densité en milieu paysan), la valeur de fanes est estimée à 125000 FCFA, alors que les $300 \mathrm{~kg}$ de grains produits, ne valent que 75000 FCFA. Les prix des fourrages varient selon les lieux et les périodes (Mahamadou, 2004 ; Manzo, 2009 ; Maman, 2014). Le marché des fanes est appelé à se développer sous l'effet de la croissance de la pratique de l'élevage et de l'embouche dans la zone agricole en général et en zones urbaines et périurbaines en particulier. Dan Gomma et al. (2000) ont noté qu'à l'occasion des fêtes de Tabaski ou en période de soudure, les prix des résidus des cultures peuvent connaître des augmentations de l'ordre de 300 à $500 \%$. Bationo et al., (2012) ont remarqué qu'en période de pénurie (mai à juin), un tas de $2 \mathrm{~kg}$ de tiges de mil ou sorgho se négociait entre 100 à 150 FCFA, alors que le même tas se vendait entre 25 à 50 FCFA en période de récolte.

à double fin (sorgho Sepon 82, niébé (LAKADE) et dolique) pour les producteurs sur le périmètre de Djirataoua car cela constitue un remède au déficit fourrager et permet aussi d'accroître la productivité du cheptel tout en procurant des revenus aux agriculteurs.

coopérative des producteurs du périmètre irrigué de Djirataoua pour avoir accepté la mise en place de l'essai sur ses parcelles.

usage double (feuilles/fourrage et graines). In : la recherche à IITA N³. Septembre 1991. 6-7

Bambara D., Zoundi J.S., Tiendrébéogo J.-P., 2008. « Association céréale/légumineuse et intégration agriculture-élevage en zone soudanosahélienne », Cahiers Agricultures, 17, (3), 297 $-301$

BATIONO B. A., KALINGANIRE A. et BAYALA J. 2012. Potentialités des ligneux dans la pratique de l'agriculture de conservation dans les zones arides et semi arides de l'Afrique de l'Ouest : 

de trois cultures : le sorgho, le niébé et la dolique à Djirataoua (Maradi - République du Niger)

Aperçu de quelques systèmes candidats. ICRAF Technical Manual no. 17, Nairobi : World Agroforestry Centre.

Camara F.S., 2010. Durabilité des systèmes pastoraux et dynamique de la strate herbacée des pâturages de la zone sahélienne du Niger (Afrique de l'ouest) Thèse : Sciences de la Nature :(Alger Faculté des Sciences Biologiques Université des Sciences et Technologies (HOUARI BOUMEDIENE).

Dabat M-H., Rabah L., Richard G., 2012. La culture du niébé au Burkina Faso : une voie d'adaptation de la petite agriculture à son environnement? Autrepart 2012, 3 (62), 95-114.

Dan Gomma A. et Rupol P., 2000. Étude sur la production des ruminants en milieu urbain et péri urbain de Niamey, AD - SV - FPI, Niamey, République du Niger. 77pp

Direction du Développement Pastoral/Direction Générale de la Promotion et des Industries Animales (DDP/DGPIA), 2015. Rapport de synthèse des résultats de la campagne pastorale 2015 2016, Ministère de l'Élevage, Niamey, République du Niger. 37pp

Hadi M., 2000. Contribution du Système d'Information sur les Marchés à Bétail (SIMB) du Niger, Conférence sur les perspectives agricoles de I'Afrique de l'Ouest (Bamako du 7 au 9 février 2000), Ministère des Ressources Animales, Niamey, République du Niger, 10pp

Hiernaux P, Diawara M. O., Kergoat L., Mougin É., 2016. La contrainte fourragère des élevages pastoraux et agro-pastoraux du Sahel, 39- 59, https://www.researchgate.net/publication/303372 607 consulté le 25 Nov. 2015

Karimou M. et Atikou A., 1998. Les systèmes agricultureélevage au Niger. In Tarawali, G. and P. Hiernaux (eds.). Improving crop. Livestock systems in the dry savannas of West and Central Africa. Reports from the Workshop on crop-livestock Systems in the dry savannas of West and Central Africa, 22.27 November 1998, Ibadan, Nigeria, 78 - 97.

Mahamadou I. M., 2004. Alimentations des ruminants en zone urbaine et périurbaine : cas de la communauté urbaine de Niamey : Rapport de stage de fin de cycle, Katibougou : IPR/IFRA. 38pp.

Mahaman I., 2001. Évolution à long terme de la fertilité de sol dans la région de Maradi, Drylands research, Working Paper 30, Crewkerne, Somerset, Royaume-Uni, 44pp.

Mahamane B., I., 2011. Étude des pratiques associées à l'élevage de la chèvre rousse de Maradi chez les femmes du département de Madarounfa, Master ès sciences Agronomiques, option Agropastoralisme, $\mathrm{N}^{\circ} 52$, Faculté d'Agronomie, Université ABDOU MOUMOUNI, Niamey, République du Niger 48pp

Maman S., 2014. Analyse du système de commercialisation du fourrage dans la ville de Niamey (Niger). Mémoire Master : Productions Animales et Développement Durable (PADD), Spécialité : Économie et Politiques d'Élevage (EPE), №7, EISMV Dakar, Sénégal, 41 pp

Manzo R.R.A., 2009. Étude du flux de fourrage vert des zones périurbaines vers la ville de Niamey en saison pluvieuse. Mémoire Sciences Agronomiques, Faculté d'Agronomie, Université Abdou Moumouni, Niamey, République du Niger, 44pp

Ministère de l'Agriculture (M/A), 2012. Catalogue national des espèces et variétés végétales (CNEV), FAO, République du Niger, 276pp.

Moussa S., 2013. Situation des sols au Niger: contraintes et besoins, Global Soil Partnership (GSP) in West Africa, 4 au 5 Février 2013 Accra, Ghana 24p.

Office National des Aménagements Hydro - Agricoles (ONAHA), 1980. Opérations de culture attelée sur les aménagements hydro-agricoles, Niamey, République du Niger.

Singh B.B., Ajeigbe H.A., Tarawali Shirley A., FernandezRivera S., Abubakar M., 2003. Improving The Production and Utilization of Cowpea as Food and Fodder, Field Crops Research, 84: 169 177.

Tarawali G., 2002. "A Synthesis of The Crop-Livestock Production Systems of The Dry Savannas of West and Central Africa", In Tarawali G., Hiernaux P.,(Editor), Improving Crop-Livestock Systems In The Dry Savannas of West and Central Africa, IITA, Ibadan, Nigeria, 23-45pp.

Van Soest P.J., 1982. Nutritional ecology of ruminant, Ithaca, New York, USA: Cornel University Press. 